\title{
Spatial Heterogeneity in Oligodendrocyte Lineage Maturation and Not Cerebral Blood Flow Predicts Fetal Ovine Periventricular White Matter Injury
}

\author{
Art Riddle, ${ }^{1}$ Ning Ling Luo, ${ }^{1}$ Mario Manese, ${ }^{1}$ Douglas J. Beardsley, ${ }^{1}$ Lisa Green, ${ }^{4}$ Dawn A. Rorvik, ${ }^{4}$ Katherine A. Kelly, ${ }^{4}$ \\ Clyde H. Barlow, ${ }^{4}$ Jeffrey J. Kelly, ${ }^{4}$ A. Roger Hohimer, ${ }^{3}$ and Stephen A. Back ${ }^{1,2}$ \\ Departments of ${ }^{1}$ Pediatrics, ${ }^{2}$ Neurology, and ${ }^{3}$ Obstetrics and Gynecology, Oregon Health and Science University, Portland, Oregon 97239-3098, and ${ }^{4}$ Barlow \\ Scientific, Inc., and The Evergreen State College, Olympia, Washington 95805
}

\begin{abstract}
Although periventricular white matter injury (PWMI) is the leading cause of chronic neurological disability and cerebral palsy in survivors of premature birth, the cellular-molecular mechanisms by which ischemia-reperfusion contributes to the pathogenesis of PWMI are not well defined. To define pathophysiologic relationships among ischemia, acute cerebral white matter damage, and vulnerable target populations, we used a global cerebral ischemia-reperfusion model in the instrumented 0.65 gestation fetal sheep. We developed a novel method to make repeated measurements of cerebral blood flow using fluorescently labeled microspheres to resolve the spatial heterogeneity of flow in situ in three-dimensional space. Basal flow in the periventricular white matter (PVWM) was significantly lower than in the cerebral cortex. During global cerebral ischemia induced by carotid occlusion, flow to all regions was reduced by nearly $90 \%$. Ischemia of 30 or $37 \mathrm{~min}$ duration generated selective graded injury to frontal and parietal PVWM, two regions of predilection for human PWMI. Injury was proportional to the duration of ischemia and increased markedly with $45 \mathrm{~min}$ of ischemia to extensively damage cortical and subcortical gray matter. Surprisingly, the distribution of PVWM damage was not uniform and not explained by heterogeneity in the degree of white matter ischemia. Rather, the extent of white matter damage coincided with the presence of a susceptible population of late oligodendrocyte progenitors. These data support that although ischemia is necessary to generate PWMI, the presence of susceptible populations of oligodendrocyte progenitors underlies regional predilection to injury.
\end{abstract}

Key words: blood flow; cerebral blood flow; hypoxia-ischemia; white matter; neonatal prenatal; oligodendrocyte; periventricular; ischemia; injury; development; blood-brain

\section{Introduction}

Periventricular white matter injury (PWMI) is a common and leading cause of chronic neurological disability in survivors of premature birth that manifests as cerebral palsy and cognitive impairment (Back and Rivkees, 2004). Two potential mechanisms for PWMI are cerebrovascular immaturity (Greisen and Borch, 2001; Volpe, 2001) and maternal-fetal infection (Hagberg et al., 2002). The timing of appearance of late oligodendrocyte progenitors (preOLs) coincides with the high-risk period for PWMI (Back et al., 2001). Early PWMI lesions display significant lipid peroxidation injury that results in pronounced degeneration of human preOLs (Haynes et al., 2003; Back et al., 2005).

\footnotetext{
Received May 4, 2005; revised Jan. 23, 2006; accepted Jan. 24, 2006.

This work was supported by National Institute of Health Grants K02 NS41343 and R01 NS045737 (S.A.B.), the Medical Research Foundation of Oregon, and the Dickinson Foundation. We are grateful for the support from Drs. Lowell Davis, John Bissonnette, and Kent Thornburg and from the Oregon Heart Center. This study is dedicated to Dr. Joseph J. Volpe on the occasion of the scientific symposium at Boston Children's Hospital to honor his leadership as the Chief of Pediatric Neurology. His relentless efforts to determine the causes of brain injury in premature infants served as the inspiration for this work.

Correspondence should be addressed to Dr. Stephen A. Back, Department of Pediatrics, Hatfield Research Center, Oregon Health and Science University, 3181 Southwest Sam Jackson Park Road, Portland, OR 97239-3098. E-mail: Backs@ohsu.edu.

D01:10.1523/JNEUROSCI.5200-05.2006

Copyright $\odot 2006$ Society for Neuroscience $\quad$ 0270-6474/06/263045-11\$15.00/0
}

These findings are consistent with a role for ischemia-reperfusion injury in the pathogenesis of PWMI. However, the extent to which susceptible preOLs define the magnitude and distribution of injury is unclear.

Current measures of global cerebral blood flow (CBF) lack the spatial resolution to define cerebral hemodynamics in human periventricular white matter. Moreover, previous experimental models have not established a relationship between PWMI and perturbations in periventricular blood flow. Small fetal and neonatal animal models have been uninformative because of a paucity of cerebral white matter, a propensity for mixed gray and white matter injury, and the technical limitations of invasive blood flow measurements (Back, 2001). In contrast, the immature ovine fetus ( 0.65 gestation) is a widely studied preparation that displays cerebral hemodynamics similar to human and permits repeated physiological measurements in utero in the unanesthetized state (Hagberg et al., 2002). The immature ovine brain is similar to preterm human between 24 and 28 weeks in terms of the completion of neurogenesis, the onset of cerebral sulcation, the detection of the cortical component of the auditory and somatosensory evoked potentials, and an increased predilection for white matter injury (Penning et al., 1994; Reddy et al., 1998). Thus, the cephalically hypotensive immature ovine fetus appears 
to be particularly susceptible to hypoxia-ischemia, but previous studies provided no data regarding blood flow to periventricular white matter.

To study the pathophysiologic relationships among ischemia, acute cerebral white matter damage, and vulnerable preOLs during prematurity, we developed a new method to repeatedly measure cerebral blood flow using fluorescently labeled microspheres to resolve the spatial heterogeneity of fetal blood flow in situ in three-dimensional (3D) space. Because preOLs are the oligodendrocyte (OL) lineage stage most susceptible to hypoxia-ischemia (Back et al., 2002b), we hypothesized that cerebral ischemia is necessary but not sufficient to cause white matter damage and that the magnitude and distribution of damage is related to the distribution of preOLs. When the immature ovine fetus was subjected to global cerebral ischemia, relatively selective graded cerebral white matter damage resulted that was proportional to the duration of ischemia. The distribution of damage was not uniform, although no significant differences were observed in white matter ischemia. Rather, the heterogeneity in distribution of white matter damage coincided with the extent to which susceptible preOLs were present. These data support that ischemia is necessary, but not sufficient, to generate PWMI and that the presence of susceptible preOLs is related to the regional predilection to injury.

\section{Materials and Methods}

Surgical procedure. This instrumented fetal cerebral hypoperfusion preparation (Reddy et al., 1998) and the general surgical procedures (Chao et al., 1991) were described previously. Time-bred sheep of mixed Western breed (88-91 d gestation; term, $145 \mathrm{~d}$ ) were studied. Twin pregnancies were studied so that each experimental fetus had a twin control. Fetuses were operated with sterile technique under general anesthesia ( $1 \%$ halothane in $\mathrm{O}_{2}$ and $\mathrm{N}_{2} \mathrm{O}$ ). Vinyl catheters were placed in the amniotic cavity and into a fetal axillary artery and fetal hindlimb vein. To confine the cerebral blood supply to the carotid arteries, the vertebro-occipital anastomoses were ligated bilaterally. These anastomoses connect the vertebral arteries, supplied by the thoracic aorta, with the external carotid arteries that are fed by the brachiocephalic (Baldwin and Bell, 1963). Hydraulic occluders (SILASTIC; Dow Corning, Midland, MI) were placed on each carotid artery. After closure of the uterus, the lines were externalized, and 1 million units of penicillin $G$ were infused into the amniotic cavity. Postoperative housing was on clean dry bedding in an individual cage with free access to food and water and close visual contact with other sheep.

Cerebral hypoperfusion studies. A total of 37 animals (see Results for the number dedicated to each experiment) were studied on the second or third postoperative day. Pressure transducers and a strip-chart recorder (TA 6000; Gould Instruments, Valley View, OH) recorded mean arterial blood pressure in the fetal artery relative to amniotic fluid pressure. Fetal heart rate was calculated from triplicate measurements of the arterial pressure pulse intervals over a continuous recording of $>20$ s. Fetuses were studied only if they demonstrated normal oxygenation $(>6 \mathrm{ml}$ $\mathrm{O}_{2} / 100 \mathrm{ml}$ blood) and blood indices, as described previously (Chao et al., 1991). Sustained cerebral hypoperfusion of 30, 37, or 45 min duration was initiated by bilateral carotid artery occlusion after inflation of the carotid occluders. Cerebral reperfusion was established by deflation of the occluders and was studied at either 15 or $60 \mathrm{~min}$ of restored flow.

Blood analysis. One milliliter blood samples taken anaerobically from the fetal axillary artery were analyzed for arterial $\mathrm{p}_{\mathrm{a}} \mathrm{H}, \mathrm{P}_{\mathrm{a}} \mathrm{O}_{2}, \mathrm{P}_{\mathrm{a}} \mathrm{CO}_{2}$ (corrected to $39^{\circ} \mathrm{C}$; IL Synthesis $10 \mathrm{pH} /$ Blood Gas Analyzer; Instrumentation Laboratory, Lexington, MA), hemoglobin content (Thb), arterial oxygen content $\left(\mathrm{CaO}_{2}\right)$, arterial oxygen saturation $\left(\mathrm{SatO}_{2}\right.$, IL $682 \mathrm{Co}$-Oximeter; Instrumentation Laboratory), and hematocrit (capillary microfuge). Fetuses were only studied if they demonstrated normal fetal oxygenation, defined as $>6 \mathrm{ml}$ $\mathrm{O}_{2} / 100 \mathrm{ml}$ of blood, at a $24 \mathrm{~h}$ recovery from the operation.

Microsphere injection protocol. Fetal brain blood flow was measured spatially by the fluorescent microsphere distribution and reference sample method (Bernard et al., 2000). Fluorescent microspheres with four different colors (15 $\mu \mathrm{m}$ diameter; F-17047, F-17048, F-17048, F-17050; Invitrogen, Eugene, $\mathrm{OR}$ ) had the following peak excitation and emission: green (450/480), yellow (515/534), red (580/605), and scarlet (650/685). Approximately $3 \times 10^{6}$ microspheres suspended in $1 \mathrm{ml}$ of saline with $0.05 \%$ Tween 20 were sonicated and then injected over $30 \mathrm{~s}$ into the fetal hindlimb vein followed by a $2 \mathrm{ml}$ flush with saline. Starting just before and continuing $2 \mathrm{~min}$ after each injection, a reference blood sample was drawn at $0.75 \mathrm{ml} / \mathrm{min}$ into a syringe mounted in a syringe pump (Harvard Apparatus, Dover, MA).

Tissue handling. At 24 or $72 \mathrm{~h}$ after cerebral reperfusion began, the ewe and fetuses were killed by intravenous injection of the ewe with Euthasol (Virbac, Ft. Worth, TX). Fetal brains were immersion fixed at $4^{\circ} \mathrm{C}$ in $4 \%$ paraformaldehyde in $0.1 \mathrm{M}$ phosphate buffer, $\mathrm{pH} 7.4$, for $2 \mathrm{~d}$ and then stored in PBS. The brains to be analyzed for microspheres were subsequently immersed in $20 \%$ sucrose until they sank and then were rapidly frozen in OCT.

Acquisition of microsphere distributions with the Imaging CryoMicrotome. Fluorescent microsphere detection and localization was performed with the Imaging CryoMicrotome (ICM; Barlow Scientific, Olympia, WA) (Kelly et al., 2000), which combines fluorescence digital imaging of frozen tissue together with serial cryostat sectioning to determine the 3D location of every microsphere. The accuracy of the ICM approach for measurement of regional blood flow versus radioactive and standard fluorescence microsphere methods has been validated (Bernard et al., 2000). The entire frozen fetal brain containing microspheres was mounted in the ICM and serially sectioned to yield $\sim 100038-\mu$ m-thick sections. Fluorescence images of the microspheres in the tissue block face were acquired at $25 \mu \mathrm{m}$ resolution. At regular intervals, adjacent sections were retrieved from the ICM for histological analysis. Fluorescent microspheres in reference blood samples were counted so that the distribution of blood flow could be related to absolute blood flow for each color. A hole drilled into a block of frozen OCT was filled with blood that had been sonicated to disperse microspheres, and the sample was frozen rapidly. An aliquot of each reference sample was serially sectioned and imaged as for the tissue samples.

Image analysis for regional blood flow. ICM-acquired fluorescence images were preprocessed to correct for variations in illumination intensity and detector response. The images were analyzed as described previously to distinguish microspheres from background tissue fluorescence and to determine the 3D location of each microsphere (Bernard et al., 2000).

Measurement of regional cerebral blood flow. Unbiased in situ measurements of blood flow were made in frontal and parietal cortex and periventricular white matter (PVWM) in a blinded manner by digital dissection of anatomically defined regions (Gluckman and Parsons, 1983; Vanderwolf and Cooley, 1990). ICM tissue block face images and the corresponding digitized images of the Nissl sections were imported to Amira 3D image analysis software (TGS, San Diego, CA). All regions were sampled in the coronal plane and contained within a region that extended $4300 \mu \mathrm{m}$ posterior to the genu of the corpus callosum (frontal blood flow) or posterior to the level of the ventral thalamic nucleus, tail of the caudate, and mammillary bodies (parietal blood flow). The 3D regions of interest (ROIs) were created by systematically outlining anatomical boundaries ascertained from coregistered Nissl sections at both extents and the middle of the sample region. The ROI volumes were $\geq 35$ $\mathrm{mm}^{3}$. Two-dimensional (2D) ROI boundaries were checked in every 10th slice for edge alignment and an interpolation algorithm applied to define volumetric ROIs encompassing the 110 slices before the incorporation of blood flow data. Hence, ROIs were defined from the histological data sets before coregistration with the microsphere data set. Therefore, the operator was masked to the blood flow data set to ensure completely unbiased data analysis. The number of microspheres within the ROIs was then determined. Blood flow was calculated from the number of microspheres in the ROI per unit volume, the reference sample withdrawal rate, and the number of microspheres in the reference sample. Blood flow is reported as milliliters per minute per $100 \mathrm{~g}$ of tissue based on a tissuespecific gravity of 1.02 .

Appropriate anatomical boundaries were systematically defined in the 

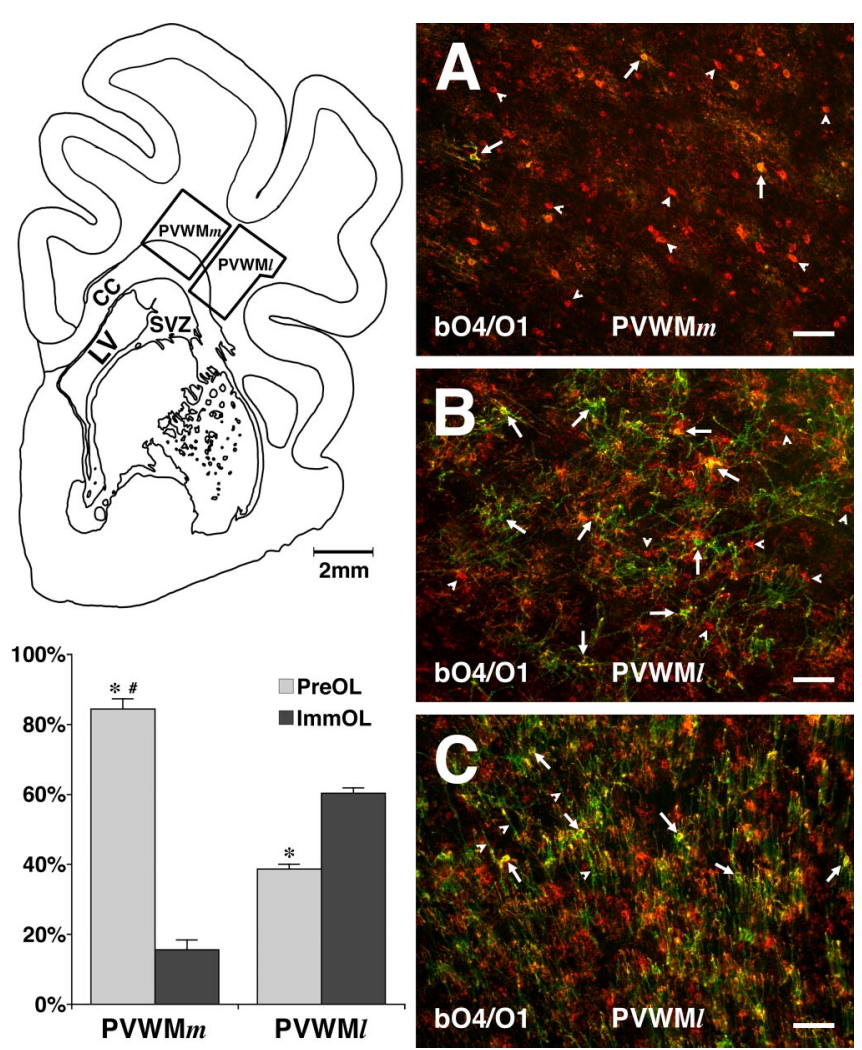

Figure 1. Spatial heterogeneity of $0 \mathrm{~L}$ maturation in 0.65 gestation ovine brain. Top left, $\mathrm{A}$ representative frontal section from the ovine brain shows schematically the approximate boundaries of the medial (PVWMm) and lateral (PVWMI) regions of the frontal PVWM. See Materials and Methods (Measurement of regional cerebral blood flow) for the definition of the boundaries of the PVWM analyzed for cell counts and blood flow measurements. A-C show the differences in OL maturation between the PVWMm and the PVWM/ by immunohistochemical double labeling with b04 (red) and 01 (green) antibodies. A, The PVWMm contains few immature $0 \mathrm{Ls}$ (arrows) $(04+, 01+)$ and numerous single-labeled pre0Ls (arrowheads) (04+, 01-). $\boldsymbol{B}$, The PVWM/ contains more differentiated cells with larger, more developed processes. The majority are immature $0 \mathrm{Ls}$ (arrows), and the minority are preOLs (red; arrowheads). $\boldsymbol{C}$, Consistent with the more mature state of the white matter in the PVWMI, numerous 01-labeled myelinated axons (arrowheads) are also seen in association with the immature 0Ls (arrows) and the smaller population of pre $0 \mathrm{Ls}$ (red). The graph (bottom left) shows the relative percentages of preOLs and immature $\mathrm{OL} s$ in the PVWMm and the PVWMI. PreOLs predominate in the PVWMm, composed of $>84 \%$ of the total OLs, and immature OLs composed the remaining $16 \%$. In the PVWMI, immature OLs are markedly increased and comprise $\sim 62 \%$ of total $0 \mathrm{Ls}$ $\left({ }^{*} p<0.001\right.$ pre 0 Ls vs immature 0 Ls for PVWM $m$ and PVWMI; ${ }^{\#} p<0.001$ pre 0 Ls in PVWMm vs PVWMI; unpaired two-tailed $t$ tests). CC, Corpus callosum; LV, lateral ventricle. Scale bars: A-C, $200 \mu \mathrm{m}$.

Nissl sections at each level. The frontal PVWM was defined as the region bounded by the apex of the lateral ventricle and three major dorsal sulci: the diagonal, ectomarginal, and cingulate sulci. Within this region, a line was drawn between the depth of the ectomarginal sulcus and the apex of the lateral ventricle that divided the PVWM into the medial and lateral PVWM. The midpoint of this line was used to define a standard region of the PVWM that was sampled in all images. This region comprised the central 50\% of the PVWM. Hence, this region did not contact the subventricular zone (SVZ), the cortical mantle, the white matter adjacent to the depths of sulci, or the superficial white matter (SWM) contained within the gyri. Parietal PVWM was defined as the region bounded by the lateral aspect of the lateral ventricle because it extends between the caudal ectosylvian sulcus and the marginal sulcus. Within this region, a line was drawn from the depth of the caudal ectosylvian sulcus and perpendicular to the lateral ventricle. The midpoint of this line was used to define a standard region that comprised the central $50 \%$ of the parietal PVWM. ROIs of the frontal cortical mantle were outlined on the dorsal surface of three primary gryi present at 0.65 gestation: the marginal, ectomarginal,
Table 1. Fetal MABP, HR, $\mathrm{p}_{\mathrm{a}} \mathrm{H}$, blood gases, arterial oxygen content, and $\mathrm{Hct} 10 \mathrm{~min}$ before ischemia (basal), at $30 \mathrm{~min}$ of ischemia, and $15 \mathrm{~min}$ after ischemia

\begin{tabular}{lccc}
\hline & Basal & Ischemia & 15 min after ischemia \\
\hline $\mathrm{HR}$ & $197 \pm 16$ & $196 \pm 23$ & $206 \pm 24$ \\
$\mathrm{MABP}$ & $36 \pm 4$ & $37 \pm 4$ & $35 \pm 4$ \\
$\mathrm{~Pa}_{\mathrm{a}} \mathrm{H}$ & $7.39 \pm 0.03$ & $7.39 \pm 0.02$ & $7.38 \pm 0.03$ \\
$\mathrm{PaC}_{2}(\mathrm{mmHg})$ & $46 \pm 3$ & $45 \pm 4$ & $47 \pm 4$ \\
$\mathrm{PaO}_{2}(\mathrm{mmHg})$ & $24 \pm 2$ & $28 \pm 3^{*}$ & $25 \pm 3$ \\
$\mathrm{HCO}(\mathrm{mmol} / \mathrm{L})$ & $28 \pm 2$ & $27 \pm 2$ & $27 \pm 2$ \\
$\mathrm{THb}_{3}^{-}(\mathrm{mmol} / \mathrm{L})$ & $10 \pm 1$ & $10 \pm 1$ & $9 \pm 1$ \\
$\mathrm{SatO}_{2} \%$ & $73 \pm 7$ & $81 \pm 5^{*}$ & $73 \pm 9$ \\
$\mathrm{CaO}_{2}\left(\mathrm{Vol} \% \mathrm{O}_{2}\right)$ & $9 \pm 2$ & $10 \pm 1^{*}$ & $9 \pm 1$ \\
$\mathrm{Hct} \%$ & $29 \pm 4$ & $30 \pm 3$ & $28 \pm 4$
\end{tabular}

Data are mean $\pm S D$. Basal, $n=33$; ischemia, $n=28 ; 15$ min after ischemia, $n=33\left({ }^{*} p<0.001\right.$ vs control; one-way ANOVA with post hoc Bonferroni for multiple comparisons). Hct, Hematocrit; HR, heart rate; MABP, mean arterial blood pressure; THb, hemoglobin content.

and rostral suprasylvian gyri. ROIs of the parietal cortical mantle were outlined on the dorsal surface of the marginal, ectomarginal, and caudal suprasylvian gyri.

Generation of 2D blood flow images. The discrete locations for fluorescent microspheres were visualized as a $2 \mathrm{D}$ spatial probability distribution using image convolution (Russ, 1999). The detailed methods can be viewed online (available at www.jneurosci.org as supplemental material).

Immunohistochemical studies. Fixed fetal brains were coronally sectioned into serial 6-mm-thick blocks of tissue with a matrix tissue slicer. Tissue blocks were serially sectioned free-floating $(50 \mu \mathrm{m})$ in PBS with a Leica (Nussloch, Germany) VTS-1000 vibrating microtome. The detailed immunohistochemical protocols to visualize specific cell types were described previously (Back et al., 2001). OL lineage cells were visualized with the $\mathrm{O} 4$ and $\mathrm{O} 1$ monoclonal antibodies. Fluorescent double labeling to distinguish preOLs $(\mathrm{O} 4+\mathrm{O} 1-)$ and immature OLs $(\mathrm{O} 4+\mathrm{O} 1+)$ used a biotinylated $\mathrm{O} 4$ antibody and the $\mathrm{O} 1$ antibody. Astroglia were visualized with a rabbit anti-bovine glial fibrillary acidic protein (GFAP) antisera (Z-0334; DAKO, Carpinteria, CA) or with a mouse anti-human vimentin antibody (Clone V9, V6630; Sigma, St. Louis, MO). Microglia and macrophages were visualized with the Bandeiria griffonia isolectin B4, biotinylated (1:100; Sigma). Axons were visualized with mouse monoclonal antibodies Sternberger Monoclonals Incorporated (SMI) 312 and SMI 31 (1:1000; Covance, Berkley, CA). Dr. Anu Srinivasan (Idun Pharmaceuticals, La Jolla, CA) generously provided the anti-activated caspase- 3 antibody (CM-1). Dr. Greg M. Cole (UCLA, Sepulveda, CA) generously supplied Fractin rabbit antisera. For fluorescent immunohistochemical studies, tissue sections were counterstained with Hoechst 33324.

Quantification of the density of TUNEL-labeled nuclei. Degenerating nuclei in frontal and parietal tissue serial adjacent sections were visualized by a terminal deoxynucleotidyl transferase-mediated biotinylated UTP nick end labeling (TUNEL) method for fluorescent in situ endlabeling of double-stranded DNA fragmentation (Back et al., 2002b). Sections were counterstained with Hoechst 33324 to delineate regional boundaries. Several strategies were done systematically to avoid introduction of counting bias. Fluorescent counterstain of nuclei was used to avoid overlapping fields. A field was not counted if it contained a tear or cerebral vessel that comprised more than $\sim 5 \%$ of the field. Cell counts were not done within fields adjacent to edges of the tissue section to avoid potential "edge artifacts," with the exception of the SVZ, where the structure is narrow and adjacent to the third ventricle. Every field containing TUNEL-labeled nuclei within the cerebral cortex, PVWM, SVZ, caudate, putamen, or hippocampus was photographed at $20 \times$ or $40 \times$ magnification [Hammamatsu (Shizuoka, Japan) Orca ER cooled CCD camera and Improvision (Lexington, MA) Openlab 4.0.2] by A.R. Between 20 and 80 images were counted by M.M. in a blinded manner in each of two adjacent sections. It should be noted that absolute stereologic methods were not feasible because of the uneven thickness inherent in the vibrating microtome-cut serial sections used for TUNEL and O4 counts and the limited penetration of antibodies into the $\sim 50-\mu$ m-thick sections (Guillery and Herrup, 1997). Free-floating sections were not subjected to 
freeze-thaw or dehydration with alcohols, which yielded optimal immunohistochemical localization of the $\mathrm{O} 4$ and $\mathrm{O} 1$ antibodies in preterm brain (Back et al., 2001). Under the ischemic conditions and survival times analyzed, there were no cystic or necrotic lesions that resulted in significant tissue distortion or shrinkage (see Results).

Generation of composite pseudocolor TUNEL maps. The distribution of cerebral lesions was plotted in the same regions from which digitized images of TUNEL-labeled nuclei were acquired. Two sections at frontal and parietal levels were analyzed in each of four animals subjected either to 30,37 , or $45 \mathrm{~min}$ of ischemia (i.e., eight sections were used to generate each composite image of the distribution of lesions for the four animals in each experimental group). The detailed methods for generation of these maps can be viewed on-line (available at www.jneurosci.org as supplemental material).

Quantification of the density of O4-labeled cells. The total density of O4-labeled cells in the medial and lateral PVWM followed the protocol, described above, for TUNEL counts. Cells were counted in a blinded manner in a minimum of three serial adjacent sections. Cell profiles that contained a nucleus, visualized with Hoechst 33324, were counted with a $20 \times$ $\left(0.125 \mathrm{~mm}^{2} /\right.$ field $)$ objective equipped with a counting grid. The profiles of both intactappearing and degenerating O4-labeled cells were counted and together comprised the calculation of total density of O4-labeled profiles. Degenerating O4-positive cells were confirmed to contain a degenerating nucleus. For each lesion, a minimum of 40 fields was counted. A minimum of 60 fields was counted for each control region.

Statistical analysis. Data analysis was performed using SPSS (Chicago, IL) 12.0 statistical software. Blood flow data were expressed as mean \pm 1 SEM. Comparisons between regions or between basal flow and occlusion were performed using paired two-tailed $t$ tests. Blood gas, hemodynamic data, and regional TUNEL indices were analyzed using ANOVA for repeated measures with post hoc inference testing done with Bonferroni or least significant difference (LSD) tests. Cell counts and time course TUNEL data were analyzed with unpaired two-tailed $t$ tests using SPSS prescribed corrections for equal or unequal variances where appropriate.

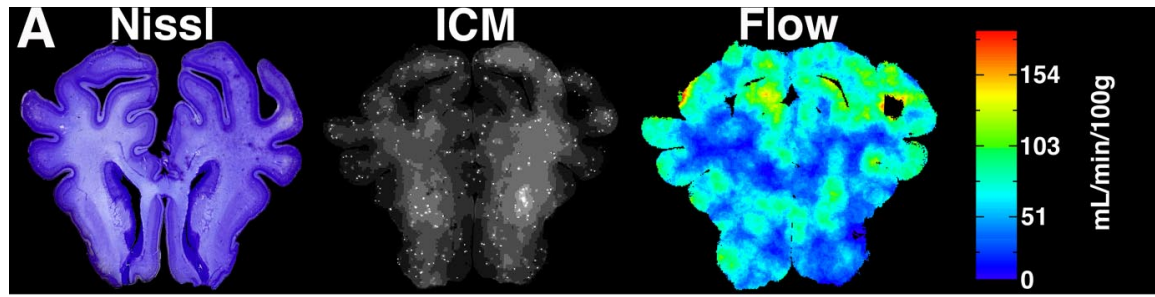

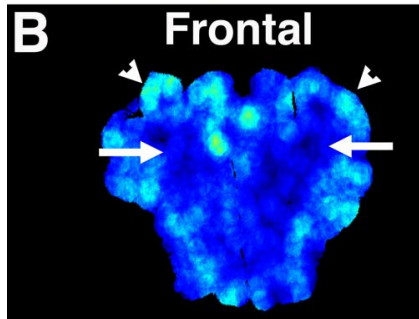

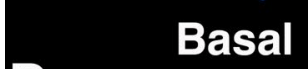

D
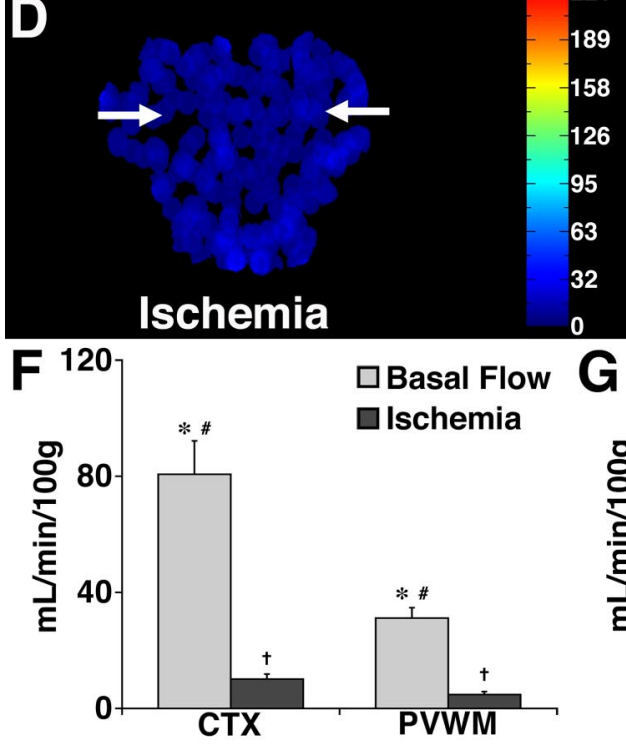

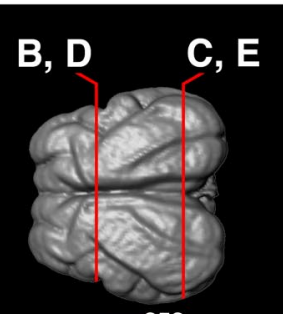

252

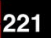

158

126

95

63 32
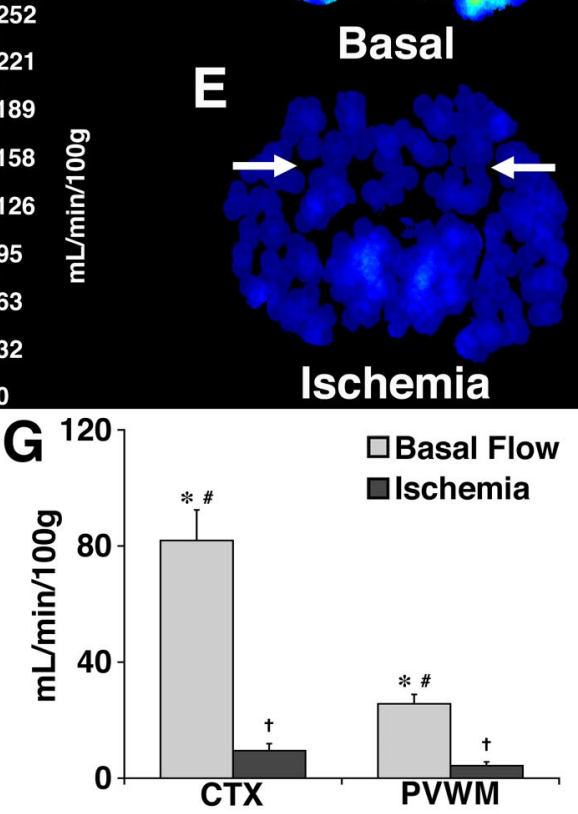

Figure 2. Quantification of fetal cerebral blood flow in situ under conditions of basal cerebral blood flow and global ischemia. $A$, Nissl-stained coronal frontal section that corresponds to the ICM image (middle) and convolved image of blood flow (right). $\boldsymbol{B}-\boldsymbol{E}$, The center image represents a 3D surface reconstruction (Amira; TGS) of magnetic resonance images of a 0.65 gestation ovine control brain that indicates the frontal and parietal levels at which the blood flow analysis was done in $\boldsymbol{B}-\boldsymbol{E}$. $\boldsymbol{B}, \boldsymbol{C}, \mathrm{Repre}-$ sentative pseudocolor basal flow images show higher blood flow (light blue) in cortical gray matter areas (arrowheads) and lower flow (dark blue) in the PVWM (arrows). The pons ( $\boldsymbol{C}$, double arrowheads) had higher basal flow rates than any region of the cerebrum. D, E, During global ischemia, blood flow was dramatically reduced in all regions and approached zero (dark blue/black) in the PVWM (arrows). $\boldsymbol{F}, \mathbf{G}$, Blood flow measured by digital dissection in the frontal $(\boldsymbol{F})$ and parietal $(\boldsymbol{G})$ cortex (CTX) and PVWM during basal blow and ischemia. ${ }^{*} p<0.001$, basal flow versus ischemia; ${ }^{*} p<0.001$, basal flow CTX versus basal flow PVWM; ${ }^{\dagger} p<0.001$, ischemia CTX versus ischemia PVWM; paired two-tailed $t$ tests.

\section{Results}

Ovine OL lineage progression at 0.65 gestation coincides with human during the high-risk period for PWMI

We first determined whether cerebral white matter maturation in the 0.65 gestation sheep is similar to that of human preterm infants at risk for PWMI. Because preOLs predominate in human PVWM during the high-risk period for PWMI (Back et al., 2001), we determined the distribution of OL lineage stages in the 0.65 gestation sheep. Figure $1 A-C$ compares the distribution of somata labeled immunofluorescently with a biotinylated $\mathrm{O} 4$ (bO4; red) and the $\mathrm{O} 1$ antibody (O1; green) in the medial and lateral frontal PVWM (PVWM $m$ and PVWM $l$ ). We studied frontal PVWM, because this is a region of particular predilection for PWMI in survivors of premature birth (Kinney and Back, 1998). We first confirmed that the total density of preOLs $(\mathrm{O} 4+\mathrm{O} 1-)$ and immature OLs $(\mathrm{O} 4+\mathrm{O} 1+)$ (defined as total O4-labeled cells) was similar in the PVWMm $(496 \pm 59$ cells $/ \mathrm{mm}^{2}$; mean $\left.\pm \mathrm{SD}\right)$ and the PVWMl $\left(471 \pm 60\right.$ cells $\left./ \mathrm{mm}^{2}\right)$. OL lineage maturation was markedly less advanced in the PVWM $m$ than in the PVWMl. PreOLs comprised $84 \pm 2 \%$ of the total O4labeled cells in the PVWMm, but in the PVWMl comprised only $39 \pm 1 \%$ (mean \pm SEM; $n=4 ; p<0.001)$. Therefore, there was approximately a threefold greater number of immature OLs in the PVWM $l$ than in the PVWMm. Parietal PVWM had less regional variation in OL lineage maturation than frontal PVWM. PreOLs comprised $68 \pm 8 \%$ of the total O4-labeled cells in the parietal PVWM. Hence, OL lineage maturation in the PVWM $m$ and parietal PVWM of the 0.65 gestation fetal sheep is similar to human preterm PVWM in that preOLs were the major OL stage present. 

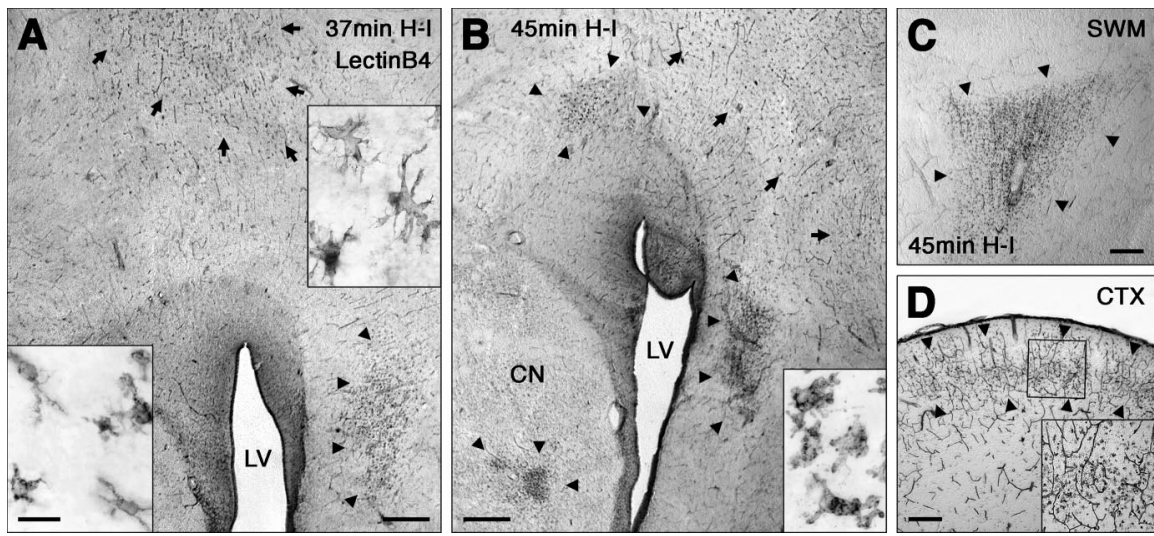

Figure 3. Histopathological features of PVWM lesions generated after global ischemia of 37 or 45 min duration, as visualized with isolectin B4.A, After 37 min of ischemia, activated microglial were localized to a lesion at the apex of the lateral ventricle (LV; arrows). The typical morphology of the activated microglia is shown in the inset at the top right. The inset at the bottom left shows the typical morphology of ramified microglia and resting macrophages in control PVWM. A focal collection of reactive macrophages/microglia localized to the lateral body of the corpus callosum (arrowheads) is shown. $\boldsymbol{B}$, After 45 min of ischemia, activated microglia were diffusely localized to a lesion at the external angle of the lateral ventricle (arrows). Focal collections of reactive phagocytic macrophages/microglia localized to medial and lateral regions in the PVWM (arrowheads). The typical morphology of the reactive phagocytic macrophages is shown in the inset at the bottom right. Note the presence of a focal lesion in the caudate nucleus (CN; arrowheads). C, D, After 45 min of ischemia, large lesions were also generated in the SWM (C, arrowheads) and in the cerebral cortex (CTX) (D, arrowheads). A detail of the cortical lesion is shown in the inset. Scale bars: $\boldsymbol{A}, \boldsymbol{B}, 500 \mu \mathrm{m} ; \boldsymbol{C}, \boldsymbol{D}, 300 \mu \mathrm{m}$; $\boldsymbol{A}, \boldsymbol{B}$, insets, $30 \mu \mathrm{m}$.

\section{Basal cerebral blood flow is low in the PVWM}

Because the developmental predilection for PWMI appears related to vascular anatomic immaturity of human PVWM (Volpe, 2001), we quantified regional cerebral blood flow by in situ analysis of fluorescent microsphere distributions. Table 1 demonstrates that under basal conditions, the fetuses exhibited normal physiological parameters (mean $\pm \mathrm{SD}$ ). By $30 \mathrm{~min}$ of ischemia, there was a small but consistent increase in $\mathrm{P}_{\mathrm{a}} \mathrm{O}_{2}, \mathrm{SatO}_{2}$, and $\mathrm{CaO}_{2}$. At 15 min after reperfusion, all blood gas and hemodynamic values returned to basal levels.

Figure 2 demonstrates our approach to obtain highresolution spatial blood flow measurements from animals subjected to $45 \mathrm{~min}$ of ischemia $(n=8)$. Figure $2 \mathrm{~A}$ shows representative data obtained after the entire fetal brain, containing fluorescent microspheres, was frozen, mounted in a cryomicrotome, and serially sectioned. A Nissl section is shown alongside a corresponding raw fluorescence ICM image that shows the location of microspheres and a reconstructed map of blood flow for the same region (Flow). Figure $2 B-E$ shows convolved maps of frontal and parietal blood flow under basal and ischemic conditions. Figure 2, $B$ and $C$, shows that basal blood flow in the frontal and parietal PVWM (arrows) was markedly lower than several adjacent regions, particularly the cerebral cortex (dorsal arrowheads) and the pons (ventral arrowheads). Figure 2, $F$ and $G$, shows data obtained by query of anatomically defined ROIs and calculation of the mean blood flow for these ROIs through post hoc digital analysis. Mean basal flow rates measured in frontal and parietal cerebral cortex were $84 \pm 13$ and $86 \pm 11 \mathrm{ml} / \mathrm{min} / 100 \mathrm{~g}$, respectively. In contrast, basal flow rates were significantly lower in the frontal and parietal PVWM $(33 \pm 4$ and $26 \pm 4 \mathrm{ml} / \mathrm{min} / 100$ g, respectively, $p<0.001)$.

\section{Global ischemia severely and proportionally reduced cerebral blood flow}

We next determined the effect of global ischemia on blood flow in selected regions of the immature ovine cerebrum. By $30 \mathrm{~min}$ of ischemia, blood flow decreased markedly throughout the brain
(Fig. 2D,E). In the frontal and parietal cerebral cortex, flow decreased significantly to $10 \pm 2 \mathrm{ml} / \mathrm{min} / 100 \mathrm{~g} \mathrm{(14} \pm 4 \%$ of basal flow; $p<0.001)$ and $10 \pm 2 \mathrm{ml} / \mathrm{min} / 100 \mathrm{~g}$ (13 $\pm 3 \% ; p<0.002$ ), respectively (Fig. $2 F, G)$. When compared with cerebral cortex, the PVWM decreased to a similarly low percentage of basal flow (frontal, $15 \pm$ $2 \%$; parietal, $16 \pm 4 \%$ ). However, the absolute flow during ischemia was significantly lower in PVWM than in cerebral cortex in both frontal $(5 \pm 1 \mathrm{ml} / \mathrm{min} / 100$ g; $p<0.001)$ and parietal $(5 \pm 1 \mathrm{ml} / \mathrm{min} /$ $100 \mathrm{~g} ; p<0.02)$ PVWM.

\section{Cerebral cortical and subcortical gray matter were less vulnerable to ischemic injury than the PVWM}

Figure 3 shows representative histopathological findings for early PVWM lesions identified at $24 \mathrm{~h}$ after an ischemic insult of 30,37 , or 45 min duration with the microglial/macrophage markers, isolectin B4 or Ham 56. Although $30 \mathrm{~min}$ of ischemia infrequently generated small focal lesions (data not shown), two types of PVWM lesions were commonly identified at 37 and 45 min (Fig. $3 A, B$ ) (supplemental Fig. 1, available at www.jneurosci.org as supplemental material). Focal lesions contained numerous reactive microglia and phagocytic macrophages visualized with both isolectin B4 and Ham 56. Diffuse white matter lesions mostly contained numerous reactive microglia. After $37 \mathrm{~min}$ of ischemia, PVWM lesions were typically more circumscribed and accompanied by limited gray matter injury. However, after $45 \mathrm{~min}$ of ischemia, PVWM lesions were more extensive and accompanied by significant lesions in the superficial white matter (Fig. $3 C$ ). In addition, large lesions in the basal ganglia (Fig. 3B) and cerebral cortex (Fig. 3D) were common.

Distribution of cerebral lesions identified by TUNEL staining To quantify cell degeneration in the PVWM, we first determined the survival time after ischemia when TUNEL staining was greater. We determined the mean density of TUNEL-labeled nuclei from frontal or parietal lesions in animals that survived for 24 or $72 \mathrm{~h}$ after 30 or $45 \mathrm{~min}$ of ischemia. Animals that survived for $24 \mathrm{~h}$ consistently had a higher mean TUNEL density in both the PVWM and cerebral cortex compared with survival for $72 \mathrm{~h}$ (supplemental Fig. 2, available at www.jneurosci.org as supplemental material).

We next generated frequency maps of the distribution of TUNEL-labeled nuclei in frontal and parietal lesions from animals that survived for $24 \mathrm{~h}$ after $30 \mathrm{~min}(n=4), 37 \mathrm{~min}(n=4)$, or $45 \min (n=4)$ of ischemia (Fig. 4$)$. In these maps, the frequency of a lesion in any location is designated by color, with no overlap in the location of lesions among animals depicted in green and the most overlap in red. After $30 \mathrm{~min}$ of ischemia, injury to frontal (Fig. 4B) and parietal (Fig. 4F) PVWM was limited and infrequently detected. After $37 \mathrm{~min}$ of ischemia, greater PVWM injury was consistently generated (Fig. 4C, G,I, yellow-to-red regions). In most animals, lesions were infrequently detected in superficial white matter, cortical gray matter, or SVZ. One exception was a predilection for neuronal injury in both frontal and parietal deep cortex. In addition, superficial 
white matter lesions occurred at the depth of the cortical gyrus adjacent to the most vulnerable region of the PVWM. Small discrete or confluent lesions of the caudate nucleus were detected in all animals. Hippocampal lesions were not detected.

After 45 min of ischemia, cerebral lesions were more extensive (Fig. 4D, $H, L$ ). Confluent lesions were commonly observed that involved both the PVWM and the superficial white matter. There was a striking increase in the extent of neuronal injury to both the cerebral cortex and the basal ganglia. Interestingly, large confluent lesions of the caudate nucleus were observed in all animals, whereas the putamen and SVZ were mostly spared. Lesions were also uncommon in the hippocampal formation (Fig. 4H). Thus, prolonged ischemia generated pronounced injury to both cerebral gray and white matter structures. However, cerebral ischemia of $37 \mathrm{~min} \mathrm{du}$ ration generated mostly selective injury to the PVWM.

Graded PVWM injury was proportional to the duration of global ischemia

We next quantified the density of TUNELlabeled nuclei in frontal PVWM lesions from animals that survived for $24 \mathrm{~h}$ after $30 \min (n=4), 37 \mathrm{~min}(n=5)$, or $45 \mathrm{~min}$ $(n=4)$ of ischemia compared with control $(n=5)$. Consistent damage of graded severity was generated between 30 and 45 min. After $30 \mathrm{~min}$, the density of TUNEL labeling in frontal PVWM was significantly increased $\left(110 \pm 13\right.$ nuclei $\left./ \mathrm{mm}^{2}\right)$ versus control ( $35 \pm 7 ; p<0.001$; one-way ANOVA with post hoc Bonferroni). The density of TUNEL-labeled nuclei in the PVWM increased to $151 \pm 17$ nuclei $/ \mathrm{mm}^{2}$ after $37 \mathrm{~min}$ of ischemia and to $294 \pm 32$ nuclei/mm ${ }^{2}$ after $45 \mathrm{~min}(p<0.05 ; 37$ vs $45 \mathrm{~min})$. Likewise, in frontal cortical lesions, mean TUNEL densities increased from $185 \pm 60$ after $37 \mathrm{~min}$ ischemia to $798 \pm 223$ nuclei/mm ${ }^{2}$ after $45 \mathrm{~min}(p<0.05$; 37 vs $45 \mathrm{~min})$. Similarly, TUNEL density in the caudate nucleus increased from $227 \pm 26$ nuclei $/ \mathrm{mm}^{2}$ at $37 \mathrm{~min}$ to $723 \pm 235 \mathrm{nuclei} / \mathrm{mm}^{2}$ at 45 $\min (p<0.05)$.

We next compared the relative vulnerability of the PVWM relative to frontal cerebral cortex, caudate nucleus, and the SVZ by analyzing TUNEL-labeled cells as a percentage of total cells in each region (Fig. 5). Relative to the other regions, the PVWM displayed the most significant injury at 30 and $37 \mathrm{~min}$ of ischemia. After $45 \mathrm{~min}$, the magnitude of injury markedly increased in a nonlinear manner, and there was a pronounced similar increase in gray and white matter injury.

\section{Differential vulnerability of glia in severe white matter lesions}

To determine the origin of degenerating nuclei in PVWM lesions from animals that survived for $24 \mathrm{~h}$, we did immunohistochem-
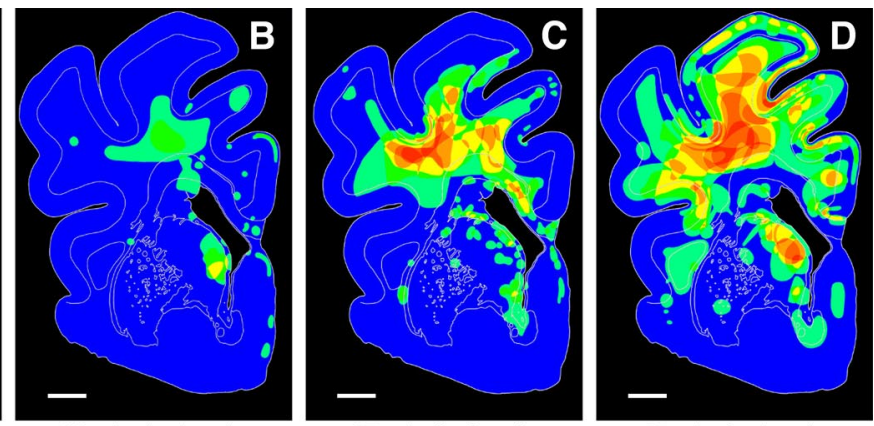

$37 \mathrm{~min}$. Ischemia
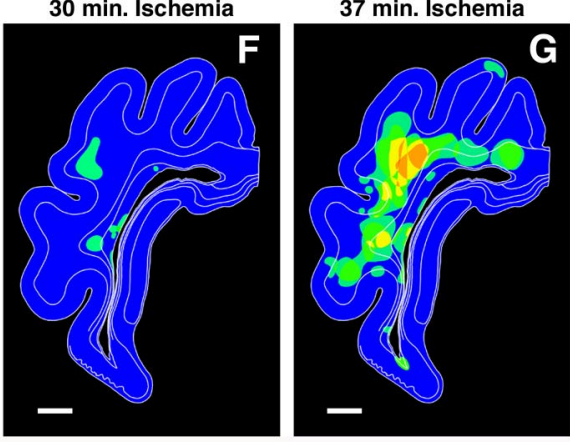

$45 \mathrm{~min}$. Ischemia
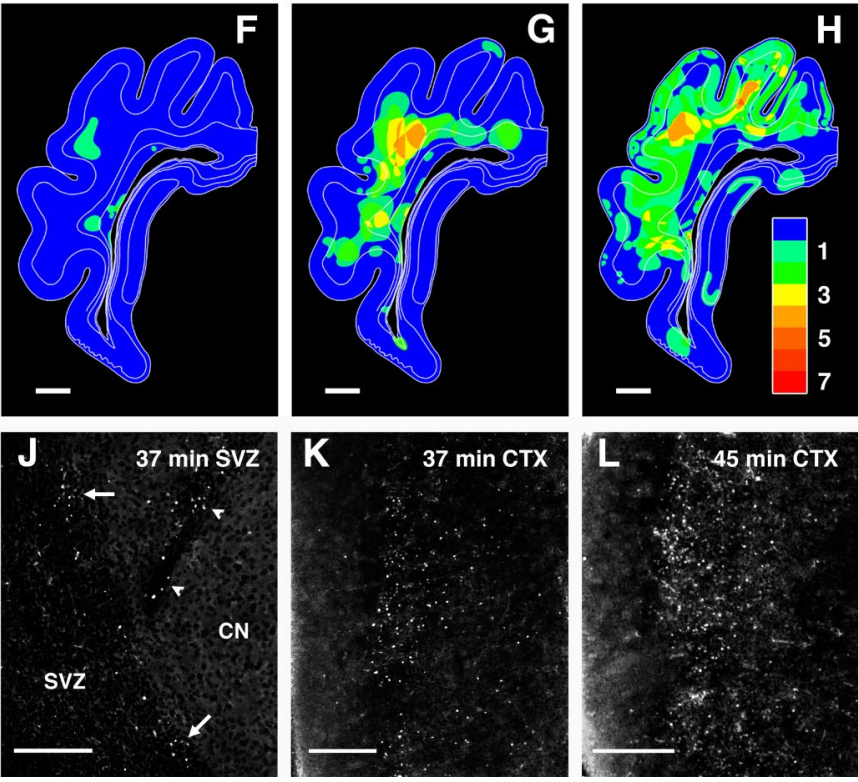

Figure 4. Regional distribution of TUNEL-labeled nuclei in cerebral lesions at $24 \mathrm{~h}$ after 30,37 , or $45 \mathrm{~min}$ of global ischemia. $\boldsymbol{A}$ and $\boldsymbol{E}$ show major anatomical structures at frontal and parietal levels of the PVWM. $\boldsymbol{B}-\boldsymbol{D}$ and $\boldsymbol{F}-\boldsymbol{H}$ show composite pseudocolor TUNEL maps of the distribution of TUNEL-labeled nuclei plotted in two sections from each of four animals. The pseudocolor that at 30 and 37 min, cerebral injury was mostly restricted to cerebral white matter. However, at 45 min, injury to the cerebral ortex and basal ganglia greatly increased, but the SVZ and hippocampus remained relatively spared. $\boldsymbol{I}-\boldsymbol{L}$ show representative examples of the magnitude and distribution of TUNEL-labeled nuclei in cerebral lesions after either $37 \mathrm{~min}(\boldsymbol{I}-\boldsymbol{K})$ or $45 \mathrm{~min}(\boldsymbol{L})$ of the SVZ, TUNEL-labeled nuclei typically localized to the dorsal extent of the SVZ (arrows) and to small extensions of the SVZ (arrowheads) into the adjacent caudate nucleus. $\boldsymbol{K}$, Focal cortical lesions were uncommon after 37 min of ischemia. $\boldsymbol{L}$, Large cortical lesions were common after 45 min of ischemia and contained a high density of TUNEL-labeled nuclei. CC, Corpus callosum; CN, caudate nucleus; CL, claustrum; EC, external capsule; HIP, hippocampus; IC, internal capsule; LV, lateral ventricle; PU, putamen. Scale bars: $\boldsymbol{A}-\boldsymbol{H}, 2 \mathrm{~mm} ; \boldsymbol{I}-\boldsymbol{L}, 200 \mu \mathrm{m}$.

ical double-labeling studies for TUNEL and cell type-specific markers. In agreement with previous studies (Falkowski et al., 2002), programmed cell death was very low throughout the control cerebral white matter. When detected, these rare TUNELlabeled nuclei typically double labeled with the O1 monoclonal antibody but not with antibodies against GFAP, vimentin, or isolectin B4.

After prolonged ischemia ( $45 \mathrm{~min} ; n=4$ ), astrocytes visualized with GFAP or vimentin appeared intact and did not double label with TUNEL (Fig. 6A,B). Similarly, microglia and reactive macrophages rarely labeled with TUNEL (Fig. 6C). As recently reported in early human PWMI lesions (Haynes et al., 2003; Back et al., 2005), degenerating astroglia and microglia were rarely detected in ovine white matter lesions after severe ischemia, which suggests that the vulnerability of these cell types is low at this time in development.

In contrast to other glial cell types, many TUNEL-labeled nu- 


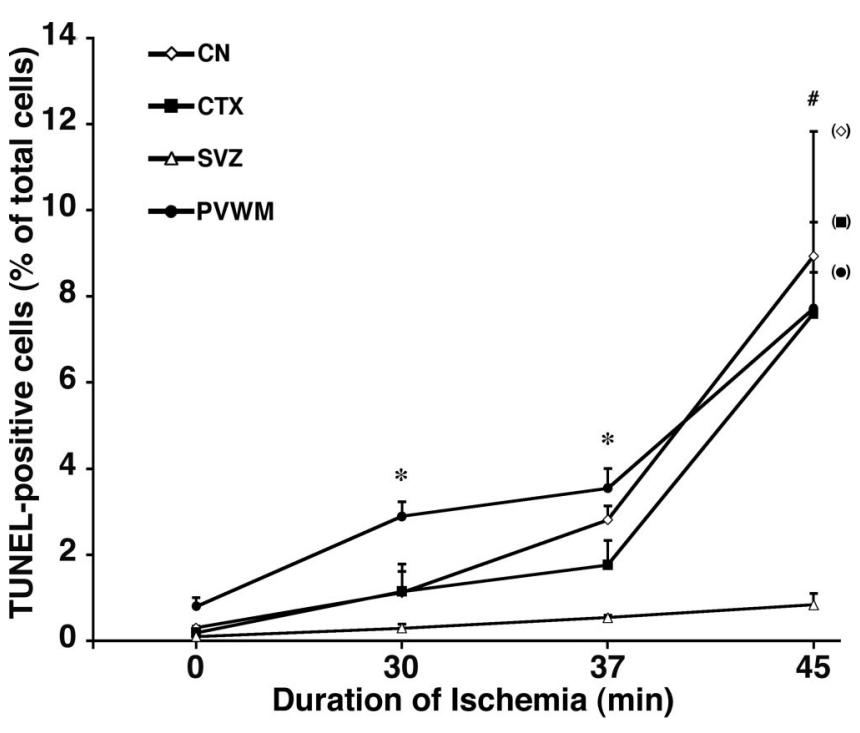

Figure 5. Regional heterogeneity of cerebral injury at $24 \mathrm{~h}$ after cerebral ischemia of 30, 37, or 45 min duration relative to control. Cell degeneration in the PVWM was compared with that in the cortex (CTX), caudate nucleus (CN), and SVZ by quantification of TUNEL-labeled cells as a percentage of total cells in each region. ${ }^{*} p<0.05$ (PVWM vs CTX; LSD test); \# indicates not significant.

clei colocalized with the $\mathrm{O} 4$ antibody, a marker of preOLs and immature OLs (Fig. 6D). The degenerating O4-labeled cells were commonly visualized in animals that sustained $37 \mathrm{~min}$ of ischemia and were also present at much lower density in mild lesions after 30 min of ischemia. Because the nuclei of these degenerating O4-labeled cells often appeared condensed or fragmented when visualized with Hoechst 33324 (Fig. 6D, inset), we determined whether some of these cells might be degenerating via a caspasedependent mechanism. We commonly visualized degenerating O4-labeled cells that double-labeled for activated caspase-3 (Fig. $6 E)$. Caspase activation was confirmed in these cells by colocalization of the $\mathrm{O} 4$ antibody with the fractin antibody that detects caspase-cleaved fragments of actin (Fig. $6 F$ ).

\section{Axonal elements were mostly resistant to ischemic injury in severe white matter lesions}

To determine the susceptibility of axons to degeneration with increasing duration of ischemia, we first visualized SMI 31, an antibody against heavily phosphorylated neurofilament proteins, together with Ham 56 to identify white matter lesions. At survival times of 24 or $72 \mathrm{~h}$, no degenerating axons were detected after 37 $\min (n=4)$ or $45 \min (n=4)$ of ischemia relative to controls in frontal cerebral white matter (Fig. 6G-I). However, using SMI 312 , an antibody used to define axonal maturation in human fetal brain (Ulfig et al., 1998; Back et al., 2002a), we detected rare degenerating axons in the PVWM of one of four animals subjected to prolonged ischemia (45 min). Degenerating axons displayed more intense staining, focal swellings, and a fragmented staining pattern (Fig. $6 \mathrm{~J}-L$ ).

\section{Differences in the OL maturational state were related to} differential susceptibility to ischemic injury in the PVWM Because OL lineage maturation was significantly less advanced in the PVWM $m$ than in the PVWMl, we hypothesized that regional maturational differences would manifest as different degrees of susceptibility to ischemia. First, we established that the overall density of degenerating cells was very similar in selective white matter lesions (PVWM $m$ and PVWM $l$ combined) when TUNEL density (151 \pm 9 ; mean $\pm \mathrm{SE} ; n=5)$ was compared with the density of degenerating O4-labeled cells $(158 \pm 21 ; n=4)$ after 37 min of ischemia. Hence, cell death in white matter lesions was mostly accounted for by the number of degenerating O4-labeled cells.

When we compared the percentage of degenerating O4labeled cells in the PVWMm (Fig. 7A,B) and PVWMl (Fig. $7 C, D)$, we found that cell death was markedly higher in the $\mathrm{PVWM} m$. In the PVWMl, many more intact-appearing cells were visualized. In fact, O4-labeled myelinating cells typically showed no features of degeneration (Fig. $7 D$, red arrows). There were approximately threefold more degenerating O4-labeled cells in the PVWMm $(27 \pm 1 \%)$ versus only $8 \pm 2 \%$ in the PVWM $l$ (Fig. $7 E)(p<0.001)$. Moreover, relative to control, there was a significant, approximately twofold greater reduction in the total density of O4-labeled cells in the PVWMm $(496 \pm 10$ vs $293 \pm 7)$ compared with the PVWMl (471 \pm 9 vs $369 \pm 6$; $p<0.01$ ) (Fig. $7 F$ ). Hence, there was a significant depletion of O4-labeled cells during the initial phase of ischemic white matter injury that was particularly pronounced in the PVWMm where preOLs predominated.

\section{Blood flow in the PVWMm and PVWMl were equivalent}

Because we found the magnitude of ischemic damage to the $\mathrm{PVWM} m$ and PVWM $l$ to differ significantly, we determined whether the difference in susceptibility of these adjacent regions might be accounted for by intrinsic differences in cerebral blood flow (Fig. 8). Under basal conditions $(n=8)$, blood flow to the PVWMm $(32 \pm 4 \mathrm{ml} / \mathrm{min} / 100 \mathrm{~g})$ and PVWMl $(33 \pm 4)$ was equivalent. During ischemia $(n=7)$, blood flow decreased similarly to $4 \pm 1 \mathrm{ml} / \mathrm{min} / 100 \mathrm{~g}$ in the PVWMm and $6 \pm 1$ in the PVWMl. Even after prolonged ischemia of 45 min duration, at both $15 \min (n=5)$ and $60 \min (n=7)$ of reperfusion, there were no significant differences in cerebral flow between these two regions. Hence, the differences in susceptibility of the PVWMm and PVWM $l$ to ischemic damage were related to differences in the maturational state of the OL lineage stages present in these adjacent regions of the PVWM.

\section{Discussion}

We developed a fetal model that yielded the following findings in support of a significant role for ischemia-reperfusion injury in PWMI: (1) graded white matter injury was generated that was proportional to the duration of ischemia; (2) selective white matter injury ranged from discrete focal lesions to diffuse injury similar to early PWMI; (3) the PVWM displayed a lower threshold for injury than cortical or subcortical gray matter or SVZ; (4) lower blood flows occurred in PVWM than in cerebral cortex but did not account for the distribution of white matter damage; (5) the distribution of white matter injury coincided with greater immaturity of the OL lineage stages present in vulnerable regions of PVWM; and (6) selective white matter injury spared most glial cell types and primarily involved premyelinating stages in the OL lineage.

We took several departures from previous approaches (Penning et al., 1994; Mallard et al., 1998; Reddy et al., 1998; Matsuda et al., 1999; Raad et al., 1999; Petersson et al., 2002) to develop a model of selective PVWM injury. To identify potential target populations of preOLs, we defined OL lineage maturation in the immature ovine brain. As in preterm human PVWM (Back et al., 2001), there were distinct regions of ovine PVWM where at-risk preOLs predominated. Second, by varying the duration of ischemia, we identified a threshold where brain injury transitioned 
from predominantly PVWM injury to extensive cerebral injury. Third, we combined cell type-specific and cell death markers to define the relative susceptibility of glia and axons under conditions of prolonged ischemia.

\section{Immature ovine ischemia reproduced histopathological features of early PWMI}

The principal pathologic features of late PWMI range from focal cystic necrosis (PVL) to diffuse myelination disturbance with gliosis (Kinney and Back, 1998). How these late lesions relate to early PWMI lesions is unclear. We recently described a range of histopathological features in human early PWMI (Back et al., 2005). The principal features were diffuse lesions in the PVWM that contained activated microglia and focal lesions rich in phagocytic macrophages. We similarly generated early ovine PVWM lesions that ranged from focal to diffuse lesions rich in microglia/macrophages. Although PWMI is often characterized by selective injury, in more severe forms it may coexist with cortical or subcortical gray and white matter injury (Kinney and Back, 1998). With more prolonged ischemia, we generated diffuse PVWM injury that was accompanied by lesions in gyral white matter, cerebral cortex, and basal ganglia. Similar lesions were detected after repeated systemic fetal endotoxin exposure (Duncan et al., 2002; Dalitz et al., 2003).

\section{Role of cerebral ischemia in PVWM injury}

In the immature fetus, shorter periods of ischemia than used here caused damage primarily to subcortical white matter (Mallard et al., 1998; Raad et al., 1999). PVWM lesions were infrequently detected in midgestation models of hypoxemia, where a restriction in uteroplacental blood flow resulted in decreased oxygen delivery and mild acidemia to the fetus without systemic hypotension or cerebral hypoperfusion (Rees et al., 1997, 1999; Mallard et al., 1998). Systemic fetal endotoxin exposure that triggered transient hypoxemia and hypotension generated superficial and PVWM lesions (Duncan et al., 2002; Dalitz et al., 2003). We found that selective white matter injury was lost with more prolonged ischemia. In fact, prolonged ischemia $(>45 \mathrm{~min}$ ) was associated with extensive cystic necrotic encephalomalacia (data not shown) as seen in severe human PVL (Marin-Padilla, 1997, 1999). Focal necrotic white matter lesions were similarly generated in response to hemorrhagic hypotension (Matsuda et al., 1999) that caused an acute decrease in cerebral blood flow of $~ 30 \%$ in a similar model (Szymonowicz et al., 1990). Hence, the neuropathological similarities of our model to human PWMI supports the notion that human $\mu \mathrm{m} ; \boldsymbol{K}, \boldsymbol{L}, 25 \mu \mathrm{m}$.
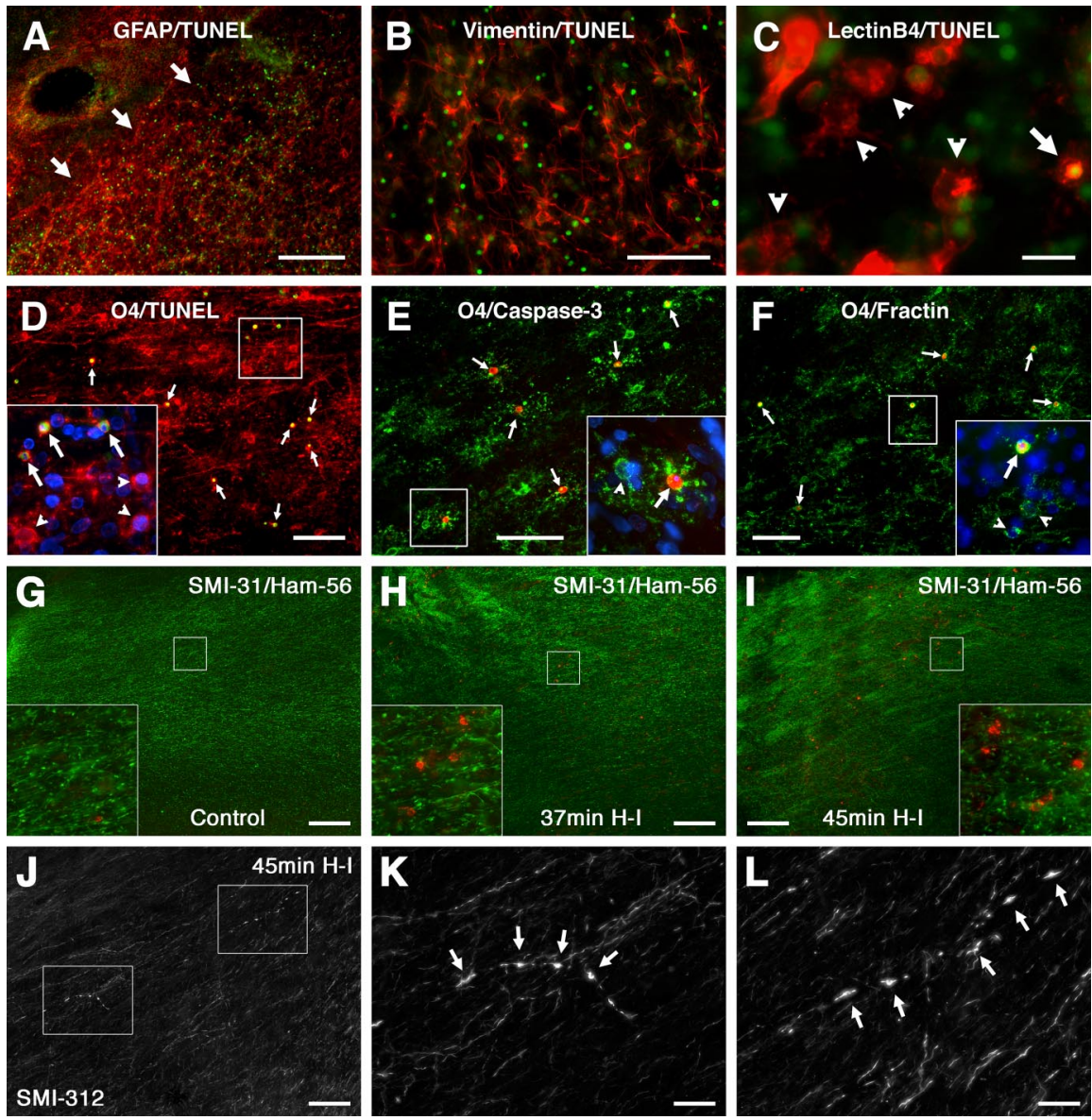

Figure 6. Differential vulnerability of glia and axons in PVWM lesions at $24 \mathrm{~h}$ after ischemia. Although astrocytes and microglia appeared mostly resistant to injury from prolonged ischemia ( $45 \mathrm{~min} ; \boldsymbol{A}-\boldsymbol{C}$ ), numerous degenerating $0 \mathrm{~L}$ precursors were detected after more mild ischemia ( $37 \mathrm{~min} ; \boldsymbol{D}-\boldsymbol{F}$ ). Axons were also mostly resistant to prolonged ischemia $(\boldsymbol{G}-\boldsymbol{L})$. $\boldsymbol{A}$, A lesion with numerous astrocytes visualized by staining for GFAP (red; arrows) that did not overlap with TUNEL-labeled nuclei (green). $\boldsymbol{B}$, A higher-power image shows intact-appearing vimentin-labeled astrocytes (red) that did not colocalize with TUNEL-labeled nuclei (green). $\boldsymbol{C}$ Most isolectin B4-labeled microglia/macrophages (red) were TUNEL negative (arrowheads), but an occasional shrunken TUNELlabeled cell appeared to be degenerating (arrow). D, A low-power image in which many degenerating 04+ cells (red) double labeled for TUNEL (green; arrows). D, Inset, High-power image shows several degenerating 04+ cells (arrows) with pyknotic 33324-labeled nuclei (blue) that also labeled with TUNEL. Note the intact cells with normal-appearing nuclei (arrowheads). $\boldsymbol{E}$, Low-power image in which many degenerating $04+$ cells (green) labeled for activated caspase-3 (red; arrows). $\boldsymbol{E}$, Inset, A triple-labeled high-power image shows a degenerating 04+ cell (arrow) that labeled for activated caspase-3 (red) and image of $04+$ cells (green) in which degenerating cells labeled with the fractin antibody (red). $\boldsymbol{F}$, Inset, A degenerating cell that labeled with fractin (arrow). Intact cells had normal-appearing nuclei (arrowheads). G-I, Low-power images of SMI 312-labeled axons (green) and Ham 56-labeled microglia/macrophages (red) in the PVWM from control (G) and animals subjected to $37 \mathrm{~min}$ $(\boldsymbol{H})$ or $45 \mathrm{~min}(\boldsymbol{I})$ of ischemia. Note the increased labeling for Ham 56 in the ischemic animals relative to control (insets) but the similar axonal staining pattern for all conditions. J, Apparent degenerating axons were rarely detected in the PVWM after profragmented staining pattern and focal swellings (arrows). Scale bars: $\boldsymbol{A}, 200 \mu \mathrm{m} ; \boldsymbol{B}, 100 \mu \mathrm{m} ; \boldsymbol{C}, 20 \mu \mathrm{m} ; \boldsymbol{D}-\boldsymbol{F}, 100 \mu \mathrm{m} ; \boldsymbol{G}-\boldsymbol{J}, 100$

PVWM may be particularly vulnerable to global cerebral hypoperfusion (Volpe, 2001).

\section{Quantification of CBF in developing brain}

We undertook an integrated analysis of CBF disturbances in regions of PVWM, where OL lineage susceptibility was defined. Even large differences in flow in small regions can be obscured when averaged with larger unaffected regions, as is the case with more global measures of CBF. This is the first quantitative study of CBF in situ achieved through digital dissection of CBF in anatomically defined regions using fluorescently labeled microspheres. Fluorescent microspheres have replaced radioactive microspheres in many 

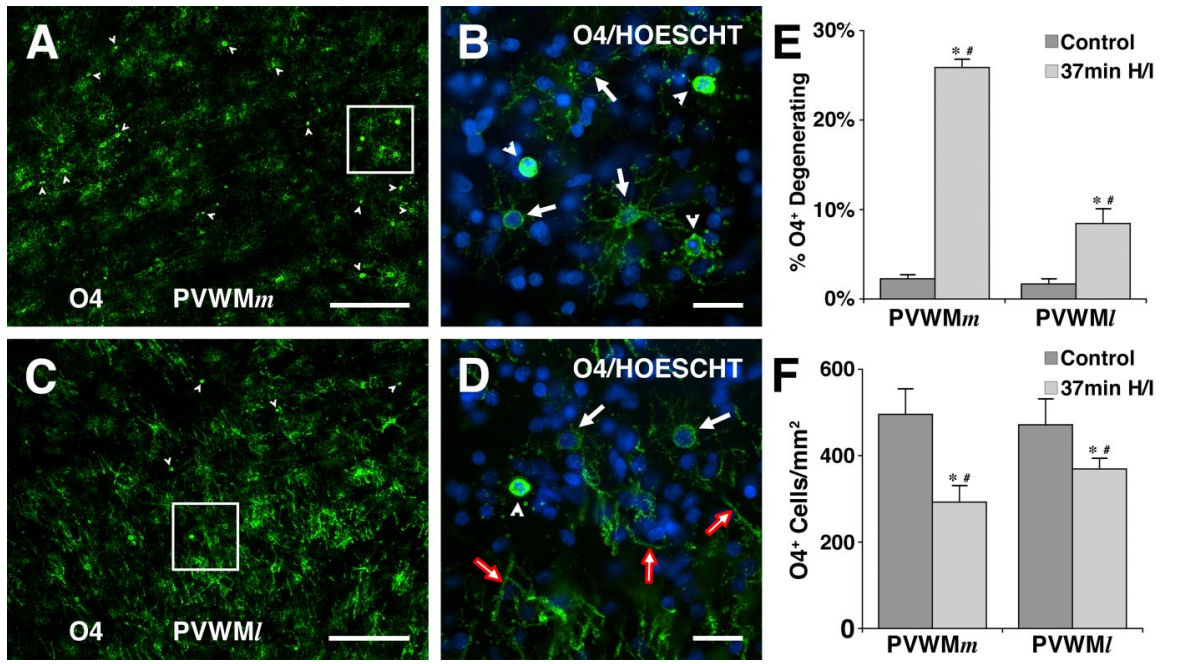

Figure 7. Differential injury in two adjacent regions of the PVWM, the PVWM $m$ and PVWM/, coincides with the extent of pre0L degeneration. $\boldsymbol{A}$, Many degenerating 04-labeled cells (arrowheads) localized to the PVWM $m$. Note the lack of early myelination in this region. $\boldsymbol{B}$, High-power image that corresponds to the inset in $\boldsymbol{A}$ shows typical degenerating cells with pyknotic Hoechstlabeled nuclei (arrowheads). Intact 04-labeled cells are indicated (arrows). C, The low-power distribution of 04-labeled cells and early myelinated axons in the PVWMI. Note that most premyelinating cells localized to the border of the myelinated tract, where occasional pyknotic cells (arrowheads) were observed. D. High-power image that corresponds to the inset in $\boldsymbol{C}$ shows a degenerating cell (arrowhead) and adjacent intact cells (arrows). Several myelinated axons are indicated (red arrows). $\boldsymbol{E}$, The percentage of total OLs degenerating at $24 \mathrm{~h}$ after ischemia. The PVWMm had a markedly higher ( $26 \pm 1 \%$ ) percentage of cells that degenerated than the PVWM/ $(8 \pm 2 \%)$. $\boldsymbol{F}$, The total density of 04 -labeled cells at $24 \mathrm{~h}$ after ischemia relative to control in the PVWM $m$ and PVWMI. Note that the total density of $0 \mathrm{~L}$ lineage cells, defined by labeling with the 04 antibody, was very similar in the control PVWMm and PVWMI. A significant loss of 04-labeled cells of 40\% occurred in the PVWMm, whereas the number of cells in the PVWM/ decreased by only $\sim 20 \%$. ${ }^{*} p<0.001$ for control versus 37 min ischemia; ${ }^{\#} p<0.01$ for ischemia PVWMm versus PVWM/; unpaired two-tailed $t$ tests. Scale bars: $\boldsymbol{A}, \boldsymbol{C}, 200 \mu \mathrm{m} ; \boldsymbol{B}, \boldsymbol{D}, 25 \mu \mathrm{m}$. Error bars represent 1 SEM.

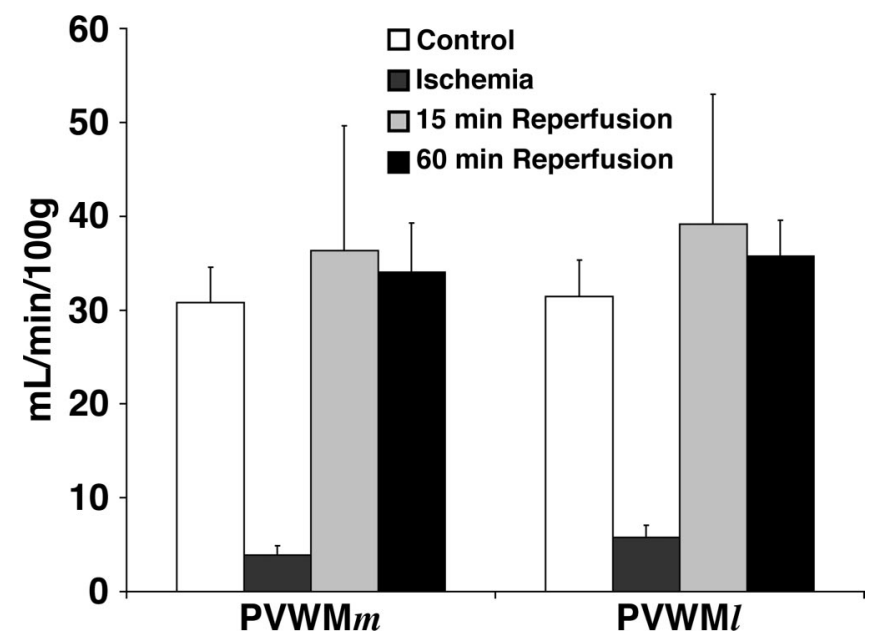

Figure 8. In situ quantification of cerebral blood flow by digital dissection of microsphere density in the PVWMm and PVWMI. No significant differences in blood flow between the PVWM $m$ and PVWM/ were detected under conditions of basal flow $(n=8)$, ischemia $(n=7)$, or at $15 \min (n=5)$ or $60 \min (n=7)$ of reperfusion. During ischemia, blood flow decreased to similar levels of $14 \pm 9 \%$ of basal in the PVWM $m$ and $17 \pm 11 \%$ in the PVWMI. After 15 min of reperfusion, there was a modest hyperemia in both the PVWMm and PVWMI. By $60 \mathrm{~min}$, blood flow returned to near basal levels again in the PVWM $m$ and PVWMI. Error bars represent 1 SEM.

laboratories, and there is a high correlation of flows determined by radio- and fluorescent-labeled microspheres (Glenny et al., 1993; Bernard et al., 2000). Under basal conditions, our blood flow measurements in white and gray matter agreed closely with those obtained with radioactive microspheres (Szymonowicz et al., 1988, 1990; Gleason et al., 1989).

Previous studies with this global hypoperfusion model in im- mature fetal sheep did not measure CBF (Reddy et al., 1998). We found PVWM flows under both basal and ischemic conditions were lower than in cerebral cortex. Although CBF in both regions fell proportionally to $\sim 15 \%$ of basal flow during ischemia, in absolute units, PVWM flow was very low relative to the cortex. Therefore, PVWM flow may have fallen below a critical threshold for hypoxic-ischemic damage, which may explain the overall greater susceptibility of PVWM relative to the better-perfused cerebral cortex.

Role of susceptible preOLs in the pathogenesis of PVWM lesions

Although global ischemia was necessary for PVWM injury, no regional differences in blood flow were found between the PVWM $m$ and PVWM $l$ under any conditions to account for the disparate rates of cellular degeneration in these regions. Our data provide the first evidence that regional differences in PVWM injury were related primarily to the maturational state of the OL lineage rather than to heterogeneity of PVWM flow. Although preOLs are particularly susceptible to hypoxiaischemia, their susceptibility in PVWM has not been addressed. Almost all cell degeneration seen in ovine PVWM lesions was accounted for by OL lineage degeneration. Conditions that produced significant preOL degeneration caused limited injury to adjacent gray matter structures. This was unexpected, because in perinatal rodents, preOL degeneration in white matter was accompanied by extensive cortical-neuronal degeneration (Back et al., 2002b) and substantial SVZ lesions (Levison et al., 2001; Plane et al., 2004). Selective degeneration of preOLs in ischemic ovine PVWM is consistent with human studies that OL progenitors are depleted in early PVWM lesions that sustain pronounced oxidative damage (Haynes et al., 2003; Back et al., 2005). Moreover, the PVWM of near-term sheep demonstrates increasing developmental resistance to ischemic injury (Penning et al., 1994; Reddy et al., 1998). Future studies are needed to define the role of acute preOL degeneration in chronic myelination disturbances. Because OL progenitors have other potential fates in gliogenesis and neurogenesis (Kondo and Raff, 2000; Belachew et al., 2003), it will be of interest to determine other potential roles of these cells in white matter regeneration and repair.

Another unexpected difference between perinatal rodents and fetal sheep is the predominant mechanism of preOL death. In a perinatal rodent model of hypoxia-ischemia, the majority of preOLs degenerated by a mechanism that did not involve apparent caspase- 3 activation (Back et al., 2002b). In contrast, ovine preOL degeneration commonly involved activation of caspase-3. Similar observations were made in late-gestation sheep (Cao et al., 2003; Castillo-Melendez et al., 2004). We speculate that the severity of the insult in our fetal model may be less than that sustained in perinatal rat models and, thus, favors a greater degree of apoptotic death. However, the relative extent of apoptotic and necrotic preOL degeneration remains to be determined. 


\section{Clinical Implications}

Although premature infants display a particular predilection for PWMI, recent studies support a widening spectrum of pathology associated with this injury that includes PVL and diffuse myelination disturbances characterized by reactive gliosis. One frequent sequela of PWMI in chronic survivors of premature birth is volume loss of cortical and deep nuclear gray matter (Inder et al., 2005). The neuropathological basis for these reductions in gray matter volume is unknown. Although small and discrete, we observed that lesions to the caudate nucleus were the most consistently observed gray matter pathology seen in association with moderate ischemia. In contrast, ovine cerebral cortex was relatively resistant to ischemia-reperfusion injury that damaged PVWM. Our data suggests that significant cortical and subcortical volume loss may arise in the setting of more severe ischemia and that there is a narrow threshold for a nonlinear increase in neuronal injury. Future studies are needed, however, to determine whether cortical or deep nuclear volume loss is related to delayed neuronal death, axonal degeneration, or depletion of subplate neurons or neural stem cells. In perinatal rats, subplate neurons are markedly more susceptible to hypoxia-ischemia than other neuronal populations (McQuillen et al., 2003), but we rarely detected degenerating cells in the ovine subplate. We also detected limited damage to SVZ progenitors.

Our data predict that those infants with earlier or more extensive OL differentiation and myelination would be less susceptible to PWMI. In fact, a more variable degree of white matter injury was detected in near-term sheep after several insults (Clapp et al., 1988; Penning et al., 1994; Mallard et al., 1998; Ikeda et al., 1999; Ohyu et al., 1999; Raad et al., 1999). Future studies are needed to determine whether acceleration of OL differentiation might be a feasible strategy to reduce the incidence and severity of PWMI.

\section{References}

Back SA (2001) Recent advances in human perinatal white matter injury. In: Prog. brain res: glial cell function (Castellano-Lopez B, Nieto-Sampedro M, eds), pp 131-147. Amsterdam: Elsevier.

Back SA, Rivkees S (2004) Emerging concepts in periventricular white matter injury. Semin Perinatol 28:405-414.

Back SA, Luo NL, Borenstein NS, Levine JM, Volpe JJ, Kinney HC (2001) Late oligodendrocyte progenitors coincide with the developmental window of vulnerability for human perinatal white matter injury. J Neurosci 21:1302-1312.

Back SA, Luo NL, Borenstein NS, Volpe JJ, Kinney HC (2002a) Arrested oligodendrocyte lineage progression during human cerebral white matter development: dissociation between the timing of progenitor differentiation and myelinogenesis. J Neuropathol Exp Neurol 61:197-211.

Back SA, Han BH, Luo NL, Chrichton CA, Tam J, Xanthoudakis S, Arvin KL, Holtzman DM (2002b) Selective vulnerability of late oligodendrocyte progenitors to hypoxia-ischemia. J Neurosci 22:455-463.

Back SA, Luo NL, Mallinson RA, O’Malley JP, Wallen LD, Frei B, Morrow JD, Petito CK, Roberts Jr CT, Murdoch GH, Montine TJ (2005) Selective vulnerability of preterm white matter to oxidative damage defined by $\mathrm{F}_{2}$-isoprostanes. Ann Neurol 58:108-120.

Baldwin B, Bell F (1963) The anatomy of the cerebral circulation of the sheep and ox. The dynamic distribution of the blood supplied by the carotid and vertebral arteries to cranial regions. J Anat (Lond) 97:203-215.

Belachew S, Chittajallu R, Aguirre A, Yuan X, Kirby M, Anderson S, Gallo V (2003) Postnatal NG2 proteoglycan-expressing progenitor cells are intrinsically multipotent and generate functional neurons. J Cell Biol 161:169-186.

Bernard SL, Ewen JR, Barlow CH, Kelly JJ, McKinney S, Frazer DA, Glenny RW (2000) High spatial resolution measurements of organ blood flow in small laboratory animals. Am J Physiol Heart Circ Physiol 279:H2043-H2052.

Cao Y, Gunn A, Bennet L, Wu D, George S, Gluckman PD, Shao X-M, Guan
J (2003) Insulin-like growth factor (IGF)-1 suppresses oligodendrocyte caspase- 3 activation and increases glial proliferation after ischemia in near-term fetal sheep. J Cereb Blood Flow Metab 23:739-747.

Castillo-Melendez M, Chow J, Walker D (2004) Lipid peroxidation, caspase-3 immunoreactivity, and pyknosis in late-gestation fetal sheep brain after umbilical cord occlusion. Pediatr Res 55:864-871.

Chao CR, Hohimer AR, Bissonnette JM (1991) Fetal cerebral blood flow and metabolism during oligemia and early postoligemic reperfusion. J Cereb Blood Flow Metab 11:416-423.

Clapp III J, Peress N, Wesley M, Mann L (1988) Brain damage after intermittent partial cord occlusion in the chronically instrumented fetal lamb. Am J Obstet Gynecol 159:504-509.

Dalitz P, Harding R, Rees S, Cock M (2003) Prolonged reductions in placental blood flow and cerebral oxygen delivery in preterm fetal sheep exposed to endotoxin: possible factors in white matter injury after acute infection. J Soc Gynecol Investig 10:283-290.

Duncan J, Cock M, Scheerlinck J, Westcott K, McLean C, Harding R, Rees S (2002) White matter injury after repeated endotoxin exposure in the preterm ovine fetus. Pediatr Res 52:941-949.

Falkowski A, Hammond R, Han V, Richardson B (2002) Apoptosis in the preterm and near term ovine fetal brain and the effect of intermittent umbilical cord occlusion. Dev Brain Res 136:165-173.

Gleason CA, Hamm C, Jones Jr MD (1989) Cerebral blood flow, oxygenation, and carbohydrate metabolism in immature fetal sheep in utero. Am J Physiol 256:R1264-R1268.

Glenny RW, Bernard S, Brinkley M (1993) Validation of fluorescent-labeled microspheres for measurement of regional organ perfusion. J Appl Physiol 74:2585-2597.

Gluckman P, Parsons Y (1983) Stereotaxic method and atlas for the ovine fetal forebrain. J Dev Physiol 5:101-128.

Greisen G, Borch K (2001) White matter injury in the preterm neonate: the role of perfusion. Dev Neurosci 23:209-212.

Guillery R, Herrup K (1997) Quantification without pontification: choosing a method for counting objects in sectioned tissue. J Comp Neurol 386:2-7.

Hagberg H, Peebles D, Mallard C (2002) Models of white matter injury: comparison of infectious, hypoxic-ischemic, and excitotoxic insults. MRDD Res Rev 8:30-38.

Haynes RL, Folkerth RD, Keefe RJ, Sung I, Swzeda LI, Rosenberg PA, Volpe JJ, Kinney HC (2003) Nitrosative and oxidative injury to premyelinating oligodendrocytes in periventricular leukomalacia. J Neuropathol Exp Neurol 62:441-450.

Ikeda T, Choi BH, Yee S, Murata Y, Quilligan EJ (1999) Oxidative stress, brain white matter damage and intrauterine asphyxia in fetal lambs. Int J Dev Neurosci 17:1-14.

Inder TE, Warfield S, Wang H, Huppi PS, Volpe JJ (2005) Abnormal cerebral structure is present at term in premature infants. Pediatrics 115:286-294.

Kelly J, Ewen J, Bernard S, Glenny R, Barlow C (2000) Regional blood flow measurements from fluorescent microsphere images using an imaging cryomicrotome. Rev Sci Instr 71:228-234.

Kinney H, Back S (1998) Human oligodendroglial development: relationship to periventricular leukomalacia. Semin Pediatr Neurol 5:180-189.

Kondo T, Raff M (2000) Oligodendrocyte precursor cells reprogrammed to become multipotent stem cells. Science 289:1754-1757.

Levison SW, Rothstein RP, Romanko MJ, Snyder MJ, Meyers RL, Vannucci SJ (2001) Hypoxia/ischemia depletes the rat perinatal subventricular zone of oligodendrocyte progenitors and neural stem cells. Dev Neurosci 23:234-247.

Mallard E, Rees S, Stringer M, Cock M, Harding R (1998) Effects of chronic placental insufficiency on brain development in fetal sheep. Pediatr Res 43:262-270.

Marin-Padilla M (1997) Developmental neuropathology and impact of perinatal brain damage. II. White matter lesions of the neocortex. J Neuropathol Exp Neurol 56:219-235.

Marin-Padilla M (1999) Developmental neuropathology and impact of perinatal brain damage. III. Gray matter lesions of the neocortex. J Neuropathol Exp Neurol 58:407-429.

Matsuda T, Okuyama K, Cho K, Hoshi N, Matsumoto Y, Kobayashi Y, Fujimoto S (1999) Induction of antenatal periventricular leukomalacia by hemorrhagic hypotension in the chronically instrumented fetal sheep. Am J Obstet Gynecol 181:725-730. 
McQuillen PS, Sheldon RA, Shatz CJ, Ferriero DM (2003) Selective vulnerability of subplate neurons after early neonatal hypoxia-ischemia. J Neurosci 23:3308-3315.

Ohyu J, Marumo G, Ozawa H, Takashima S, Nakajima K, Kohsaka S, Hamai Y, Machida Y, Kobayashi K, Ryo E, Baba K, Kozuma S, Okai T, Taketani Y (1999) Early axonal and glial pathology in fetal sheep brains with leukomalacia induced by repeated umbilical cord occlusion. Brain Dev 21:248-252.

Penning D, Grafe J, Hammond R, Matsuda Y, Patrick J, Richardson B (1994) Neuropathology of the near-term and midgestation ovine fetal brain after sustained in utero hypoxemia. Am J Obstet Gynecol 170:1425-1432.

Petersson K, Pinar H, Stopa E, Faris R, Sadowska G, Hanumara R, Stonestreet B (2002) White matter injury after cerebral ischemia in ovine fetuses. Pediatr Res 51:768-776.

Plane J, Liu R, Wang T, Silverstein F, Parent J (2004) Neonatal hypoxicischemic injury increases forebrain subventricular zone neurogenesis in the mouse. Neurobiol Dis 16:585-595.

Raad RA, Tan WK, Bennet L, Gunn AJ, Davis SL, Gluckman PD, Johnston BM, Williams CE (1999) Role of the cerebrovascular and metabolic responses in the delayed phases of injury after transient cerebral ischemia in fetal sheep. Stroke 30:2735-2741.

Reddy K, Mallard C, Guan J, Marks K, Bennet L, Gunning M, Gunn A, Gluckman P, Williams C (1998) Maturational change in the cortical response to hypoperfusion injury in the fetal sheep. Pediatr Res 43:674-682.

Rees S, Stringer M, Just Y, Hooper S, Harding R (1997) The vulnerability of the fetal sheep brain to hypoxemia at mid-gestation. Dev Brain Res 103:103-118.

Rees S, Breen S, Loeliger M, McCrabb G, Harding R (1999) Hypoxemia near mid-gestation has long-term effects on fetal brain development. J Neuropathol Exp Neurol 58:932-945.

Russ J (1999) Image processing handbook. Boca Raton, FL: CRC LLC.

Szymonowicz W, Walker AM, Cussen L, Cannata J, Yu VY (1988) Developmental changes in regional cerebral blood flow in fetal and newborn lambs. Am J Physiol 254:H52-H58.

Szymonowicz W, Walker A, Yu V, Stewart M, Cannata J, Cussen L (1990) Regional cerebral blood flow after hemorrhagic hypotension in the preterm, near-term, and newborn lamb. Pediatr Res 28:361-366.

Ulfig N, Nickel J, Bohl J (1998) Monoclonal antibodies SMI 311 and SMI 312 as tools to investigate the maturation of nerve cells and axonal patterns in human fetal brain. Cell Tissue Res 291:433-443.

Vanderwolf C, Cooley R (1990) The sheep brain: a photographic series, Ed 2. London, Ontario, Canada: A. J. Kirby.

Volpe JJ (2001) Neurobiology of periventricular leukomalacia in the premature infant. Pediatr Res 50:553-562. 